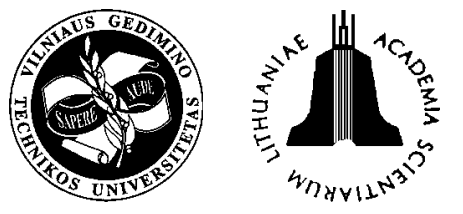

ISSN 1648-4142 TRANSPORT

\title{
MODELLING OF FREIGHT FLOWS DISTRIBUTION AT THE TRANSPORT TERMINAL
}

\author{
Adolfas Baublys \\ Transport Research Institute, Vilnius Gediminas Technical University, Plytinès g. 27, LT-10105 Vilnius-16, Lithuania, \\ E-mail: abaublys@takas.lt
}

Received 2003-07-30; accepted 2004-02-17

\begin{abstract}
The task model has been formulated for users of freight flows at a distribution transport terminal, which enables gauging vehicles possibilities (dimensions, carrying capacity), delivery time limitations, resources of vehicle time and general costs.

The synthesis of the topological structure of the freight road transport regional system has been performed. It enables the evaluation of an impact on the functioning of the system exercised by various structural system characteristics, thus improving the quality of project solutions at the stage of synthesis of the system structure.
\end{abstract}

Keywords: freight road transport; synthesis of the topological structure.

\section{Introduction}

Goods from the transport terminal have to be distributed to different consumers by small consignments, which cannot be disjointed, however, they can be transported together with other consignments. The goods from the terminal have to be carried with the view of satisfying the consumer's demands and efficient use of vehicles.

The improvement of long distance freight transport and transportation of 20-40 $\mathrm{t}$ freight by vehicles has been analysed [1-6]. However there is practically no research into the transportation of $50-1500 \mathrm{~kg}$ freight in the transport network spread in a small locality, such as, for example, a town, where the network is clogged by vehicle congestions and ecological restrictions are introduced. Therefore, certain solutions will be proposed in this research work.

\section{Model of freight flows formation}

Let $M$ be a fixed number of vehicles used, $N-$ a fixed number of freight delivery points; $I_{M}-$ $\{1,2, \ldots, M\}$ a set of indices of all transport facilities, with the particular vehicles further indexed by $I ; I_{N}-$ $\{1,2, \ldots, N\}$ a set of indices of freight delivery points, with the particular points further indexed by $j$. Any transport facility $i\left(i \in I_{M}\right)$ is given: $G_{(i)}$ - carrying capacity; $T_{(i)}$ - time resource (planned period of transportation); $D_{(i, k)}$ - size $k$ of the cargo section of vehicle $\left(k \in K_{L}=\{1, \ldots, L\}\right.$; here, $L-$ a number of sections of various carrying capacities.
It should also be noted that a consignment of goods to be delivered to point $j$ is characterized by the following parameters: $\left(j \in I_{N}\right) ; g(j)$ - freight weight; $d(j, k)$ - freight size $k ;\left(k \in K_{L}\right) ; T_{(j)}$-specified delivery time. It is assumed that each consignment of goods should be delivered to the particular point, while several consignments to be delivered to the same point are combined together, therefore, each point $j$ may be associated with the consignments of goods $j$.

It may be stated that the average speed of a vehicle carrying goods between the points $j_{1}$ and $j_{2}$ depends on the profile of the road as well as on vehicle index $i$ and weight of freight $G$. Then, the average speed of transportation will depend on parameters $j_{1}, j_{2}, i$ and $G$ and will be denoted further by $\bar{u}\left(j_{1}, j_{2}, i, G\right)$.

A matrix of distances $A$ between the points of each pair $\left(j_{1}, j_{2}\right)$ is known. Relying on the distance $a\left(j_{1}, j_{2}\right)$ between the points of the pair $\left(j_{1}, j_{2}\right)$ and the average speed of vehicle between the above points $\bar{U}\left(j_{1}, j_{2}, i, G\right)$, it is possible to determine the time of freight carriage from point $j_{1}$ to point $j_{2}$ for any vehicle $i$ :

$$
t\left(j_{1}, j_{2}, i, G\right)=a\left(j_{1}, j_{2}\right) / \bar{U}\left(j_{1}, j_{2}, i, G\right) .
$$

Let us formalize a series of transportation routes aimed to comprise all points or a set of cargoes to be delivered. Let us also assume that transport facilities are at the terminal, which will be assigned an index $(N+1)$. Then, let us denote by $a(j, N+1)$ and $a(j, N+1, j)$ the distances from the point $j$ to the point $(N+1)$ and from $(N+1)$ to $j$, respectively.

A series of routes will be characterized by the vec- 
tors $x, y$ of integer numbers and the rearrangement $\pi=\left(\pi_{1}, \pi_{2}, \ldots, \pi_{N}\right)$ of the elements of the set $I_{N}$ (i.e. the rearrangement of indices of the delivery points). Let us assume that the vectors $x$ belong to the sets $X(m)$, here, $m-M$ is a size vector, with the components $m_{i}$ and

$$
X(m)=\left\{x \in E^{M} \mid 1 \leq x_{i} \leq m_{i}, i \in I_{m}\right\},
$$

here and further, $E^{q}$ denotes a set of size vectors of all numbers $q$.

The vectors $y$ belong to the sets $Y(|x|)$, here,

$$
\begin{aligned}
& |x|=\sum_{i \in{ }^{I} M} x_{i} \text { and } \\
& Y(|x|)=\left\{y \in E^{|x|}=y_{1}<y_{2}<\ldots<y_{|x|}<N\right\} .
\end{aligned}
$$

Physical meaning of the vectors introduced is as follows: the component $x_{i}$ of the vector $x(i=1,2, \ldots, M)$ denotes a number of hauls for the vehicle $i$ (while $m_{i}$ is a prescribed estimate of the above number); the component $y_{l}$ of $y(l=1,2, \ldots,|x| x \mid)$ denotes the first position of the route $l$. More exactly, the total of vectors $x \in X(m), y \in Y(|x|)$ and rearrangements $\pi$ determine the routes $|x| x \mid$, while each $l$ from $\pi_{l}$ is expressed in the following way:

$$
\Pi_{l}=\left(N+1, \pi_{y_{l}}, \pi_{y_{l+1}}, \ldots, \pi_{y_{l+1}-1}, N+1\right) .
$$

All the routes $(u)$ are allotted to $M$ groups by the components of the vector $x$.

Each group of routes $i$ refers to vehicle $i$, while the numbers of routes of this group belong to the interval $\left(\bar{x}_{i}, \bar{x}_{i+1}\right)$; here, the values $\bar{x}$ may be obtained via the components of the vectors $x$ in the following way:

$$
\bar{x}_{1}=1, \bar{x}_{i+1}=\bar{x}_{i}+x, i=1,2, \ldots, M .
$$

Let us denote each route $l$ by $(l=1,2, \ldots,|x|)$ :

$$
\begin{aligned}
& G\left(\Pi_{l}\right)=\sum_{r=y_{l}}^{y_{l+1}-1} g\left(\pi_{r}\right), \quad G\left(\Pi_{l}, q\right)=G\left(\Pi_{l}\right)-\sum_{r=y_{l}}^{y_{l+q}} g\left(\pi_{r}\right), \\
& q=1,2, \ldots,\left(y_{l+1}-y_{l}\right)-1 .
\end{aligned}
$$

Let us set the constraints on a system of routes and their allotment to the particular transport facilities. The limitations are also distributed among the groups similarly to route distribution among the vehicles. Let the route $l$ belong to group $i$, i.e. $l \in\left[\bar{x}_{i}, \bar{x}_{i+1}\right)$, here $i \in I_{M}$.

First, the constraint on the total freight weight is imposed on this route, implying that the above value cannot exceed carrying capacity of the vehicle $i$ :

$$
G\left(\Pi_{l}\right) \leq G(i) .
$$

Secondly, to the route $l$ a constraint is applied for overall freight dimensions, which cannot exceed the dimensions of cargo section of the vehicle $i$ :

$$
D_{k}\left(\Pi_{l}\right) \leq D(i, k), k \in K_{L} \text {. }
$$

The notation $D_{k}\left(\Pi_{l}\right)$ is similar to that introduced to denote the first relationship in the expression (6).

Third, time limits are imposed on the route $l$ :

$$
\begin{aligned}
& t\left[N+1, \pi_{r_{l}}, i, G\left(\Pi_{l}\right)\right] \leq T\left(\pi_{r_{l}}\right), \\
& t\left[N+1, \pi_{r_{l}}, i, G\left(\pi_{l}\right)\right]+\sum_{q=r_{l}}^{\bar{q}} t\left[\pi_{q}, \pi_{q+1}, i, G_{q}\left(\Pi_{l}\right)\right] \leq T\left(\pi_{\bar{q}}\right), \\
& \quad \bar{q}=r_{l}+1, r_{l}+2, \ldots, r_{l+1}-1 .
\end{aligned}
$$

Time limits are imposed on the total of the routes:

$$
\begin{aligned}
& \sum_{l=\bar{x}_{i}}^{x_{i+1}-1}\left\{t\left[N+1, \pi_{r_{l}}, i, G\left(\Pi_{l}\right)\right]+t\left[\pi_{r_{l+1}-1}, N+1, i, 0\right]+\right. \\
& \left.\sum_{l=r_{l}}^{\bar{x}_{i+1}-1} t\left[\pi_{q}, \pi_{q+1}, i, G_{q}\left(\Pi_{l}\right)\right]\right\} \leq T(i)
\end{aligned}
$$

in expressions (9) and (10) the notation found in (1) and (6) is used.

Thus, overall costs $Z(x, y, \pi)$ depending on the system of routes and their distribution among the particular facilities defined by the vectors $x, y$ and rearrangement $\pi$ may be obtained as follows:

$$
\begin{aligned}
& Z(x, y, \pi)=\sum_{i=1}^{M} \sum_{l=\bar{x}_{i}}^{\bar{x}_{i+1}-1}\left\{Z\left[N+1, \pi_{r_{l}}, i, G\left(\Pi_{l}\right)\right]+\right. \\
& \left.Z\left[\pi_{r_{l+1}-1}, N+1, i, 0\right]+\right\} \\
& \sum_{l=r_{l}}^{\bar{x}_{i+1}-1} Z\left[\pi_{q}, \pi_{q+1}, i, G_{q}\left(\Pi_{l}\right)\right] .
\end{aligned}
$$

In the equation (11), the costs of carrying the freight of the weight $G$ from the point $j_{1}$ to the point $j_{2}$ by vehicle $i$, are denoted by $Z\left(j_{1}, j_{2}, i, G\right)$. Generally, the costs may be expressed in terms of the distance $a\left(j_{1}, j_{2}\right)$ between the points $j_{1}$ and $j_{2}$ or by multiplying the above distance by all carried goods. In other cases, the relationship between the costs considered and the parameters $j_{1}, j_{2}, i$, and $G$ may be more complicated, for example, if the costs are determined in terms of the fuel used.

In transportation various types of costs should be taken into account. Therefore, the model considered is aimed to embrace various costs, denoting them by the index $S$ and determining them as shown in the equation (11). Thus, let us determine the costs $(S+1)$ of the type:

$$
Z^{(s)}(x, y, \pi) \text {, here } s=0,1,2, \ldots, S \text {. }
$$

Then, let $Z^{(0)}(x, y, \pi)$ be overall costs to be minimized, while other kinds of expenses may be at the high- 
est admissible level $Z(s)$. Then one more group of limitations referring to the overall costs will be added to the previously formulated constraints:

$$
Z^{(s)}(x, y, \pi) \leq Z(s), s=1,2, \ldots, S,
$$

here $Z^{(s)}(x, y, \pi)$ is found based on the values $Z^{(s)}\left(j_{1}, j_{2}, i, G\right)$ according to the formula (11), while the values $Z\left(j_{1}, j_{2}, i, G\right)$ are used to find overall costs $Z(x, y, \pi)$. Now, the problem associated with the flows of consignments of goods between the terminal and customers may be formulated:

to find

$$
\min _{x \in X(m)} \min _{y \in Y(|x|)} \min _{\pi \in \Pi_{N}}\left\{Z^{(0)}(x, y, \pi)\right\}
$$

with the limitations (7)-(10), (12).

\section{Synthesis of the topological structure of a road freight transport regional system}

In designing a logistics centre of transport distribution the topological structure of the area, which is serviced by this centre, should be worked out. In the above structure a number of the local warehouses, their capacity and location in the zone as well as the settlements (or the particular urban areas), which will be serviced by the particular warehouses, for instance, by those belonging to a commercial enterprise "MAXIMA", should be provided for.

For example, the network of the settlement (town) roads for freight transportation is expressed by the matrix $D=\left\|d_{i i^{\prime}}\right\|$, where $d_{i i^{\prime}}$ are the shortest routes in the road network between the $i$-th and the $i^{\prime}$-th points; $d_{i i^{\prime}}=d_{i^{\prime} i}$. The amount of goods to be carried in the particular region serviced by the particular company is also determined by the matrix $A^{K}=\left\|a_{i i^{\prime}}\right\|$, where $a_{i i^{\prime}}$ is the flow of goods from the point $i$ to the point $i^{\prime}$. The purpose of the topological synthesis of a freight road transport system comprising a set of commercial enterprises in the region $E^{n}$, the route between the points $D$ and the flow of goods $A^{k}$, is to divide the settlements of a region into zones serviced by the warehouses $E_{j}\left(\cup E_{j}=E^{n}, E_{j} \cap E_{j^{\prime}}=\varnothing\right)$ and to determine the location of warehouses within zones $E_{t}$, minimizing the costs of goods transportation in the region. This is expressed in the following way:

$$
\begin{aligned}
& \sum_{j \in J} \sum_{i \in I i^{\prime} \in I} c_{1} d_{i i^{\prime}} x_{i j} x_{i j^{\prime}}+ \\
& \sum_{j \in J} \sum_{j^{\prime} \in J} \sum_{k \in I} \sum_{k^{\prime} \in I} c_{2} d_{k k^{\prime}} y_{k j} y_{k^{\prime} j^{\prime}}+\sum_{i \in I i^{\prime} \in I} a_{i i^{\prime}} x_{i j} x_{i j^{\prime} j^{\prime}}+ \\
& \sum_{j \in J} \sum_{j^{\prime} \in J} \sum_{k \in I} \sum_{k^{\prime} \in I_{j}} c_{1} d_{k k^{\prime}} y_{k^{\prime} j}+\sum_{i \in I_{j}} \sum_{i^{\prime} \in I_{j}} a_{i i^{\prime}} x_{i j} x_{i j^{\prime}},
\end{aligned}
$$

here $x_{i j}=1$ if the point $i$ enters the zone $j$ of the warehouse; in the opposite case $x_{i j}=0 ; y_{i j}=1$, if the warehouse is located at the point $i$; for the opposite case - $y_{i j}=0 ;$ - cost of goods transportation by low or medium functional capacity vehicles; $c_{2}$ - cost of transportation by high functional capacity lorries and trailers; $J$ - a set of warehouse indices; $I_{j}-$ a set of indices of settlements in the $j$-th warehouse zone; $N-$ a set of indices of the region settlements where warehouses may be located.

The first term in the formula (14) expresses the cost of goods transportation within a zone, while the 2-nd term denotes the cost of goods transportation from one warehouse to another, and the $3-\mathrm{d}-$ the cost of goods carriage to and from the warehouse.

The synthesis of the topological structure of freight road transport regional system (FRTRS - KKTRS) is performed by solving two problems:

a) the distribution of a set of points supplying and receiving goods according to zones serviced by the particular warehouses, minimizing the total freight flows disbalance between the warehouses;

b) determining the location of the warehouse within a zone, minimizing the total volume of work of delivering goods to a warehouse and taking them out of it. The total disbalance of cross-hauls will be the optimisation criterion of the problem $a$, because this is the main cause why the functional capacity of vehicles and the hauls are not used to the full advantage.

Let $P_{i}=\left\{E_{i / j}=\overline{1,2^{n}}\right\}$ be a family of all $E_{j}$ subsets of a region set of points $E^{P}=\{1,2, \ldots, i, \ldots, n\}$. Here, $n$ is the number of points in the region. In order to explain the $k$-th expansion of the set $E^{P}$, let us introduce the set $J_{k}$ which is such a set with the index $E_{j}$ that $\bigcup_{j \in J_{k}}=E^{P}, E_{j} \cap E_{j^{\prime}}=\varnothing, V_{j}, j^{\prime} \in J_{K}$. Then, the $k$-th expansion of the set $E^{P}$ is such a subfamily $P_{k}\left(P_{k} \subset R\right)$ that $P_{k}=\left\{E_{j} / j \in J_{k}\right\}$, where $J_{k}$ means a subset $E_{j}$ of the expansion. The disbalance of crossflows of goods of the warehouse of the zone serviced by the warehouse $j$ will be as follows:

$$
\alpha_{j}=\left|\sum_{i \in E_{j}} \sum_{i^{\prime}=E_{j}}\left(a_{i i^{\prime}}-a_{i^{\prime} i}\right)\right|,
$$

here $a_{i i^{\prime}}$ is the flow of goods from the point $i$ to the point $i^{\prime}$.

The total disbalance among the warehouses in the system for the $k$-th expansion is determined in the following way:

$$
z=\sum_{j \in J} \alpha_{j} x_{j}
$$

here $x=\left\{\begin{array}{l}1, \text { if } j \in J_{k}, \\ 0, \text { if } j \notin J_{k},\end{array}\right.$ 
here, $J$ is a set of indices of the subset $E_{j}$, the potential zones serviced by warehouses.

Formalizing the problem of the system structure synthesis, the requirements to structural and functional parameters are stated by the constraints of two types: 1) the constants of structural parameters of the system are commonly expressed by the analytical expression; 2) algorithmic expression is mostly used for functional parameters requirements (the sophisticated relationships of these parameters can not be expressed analytically).

The following analytically expressed constraints can be suggested:

The requirements that the subsets do not cross each other and cover the whole sets of the region points:

$$
\begin{gathered}
\sum_{j \in J} t_{i j} x_{j}=1, i \in E^{P}, \\
\text { here } t_{i j}=\left\{\begin{array}{l}
1, \text { if } i \in E_{j}, \\
0, \text { if } i \notin E_{j} .
\end{array}\right.
\end{gathered}
$$

The number of the region warehouses serviced is:

$$
Q_{\min }^{t} \leq \sum_{j \in J} x_{j} \leq Q_{\max }^{t},
$$

here $Q_{\min }^{t}, Q_{\max }^{t}$ - permissible and maximum numbers of warehouse zones in the system for the warehouse number in each zone:

$$
\sum_{i \in E^{P}} b_{i} t_{i j} \geq 1, \quad j \in J
$$

here

$$
\begin{gathered}
b_{i}=\left\{\begin{array}{l}
1, \text { if a warehouse may be set up in point } i, \\
0-\text { for the opposite case; }
\end{array}\right. \\
G_{\min }^{l} \leq \sum_{i \in E^{P}} t_{i j} \leq G_{\max }^{l}, \quad j \in J,
\end{gathered}
$$

here $G_{\min }^{l}, G_{\max }^{l}$-permissible minimum and maximum numbers of points in a warehouse zone:

$$
d_{i i} t_{i j} t_{i^{\prime} j} \leq L^{l}, \forall i, i^{\prime} \in E^{P}, j \in J,
$$

for the length of the route between the warehouse $j$ and the points of its zone:

$$
d_{i i^{\prime}} \leq L_{T}^{l}, \quad \forall i^{\prime}: b_{i^{\prime} j}=1, \quad j \in J, \quad i \in E^{P},
$$

here $L_{T}^{l}$ is the permissible length of the route between the warehouse $j$ and the points of its zone; the total volume of goods in a flow handled in the warehouse $j$ :

$$
\sum_{i \in E^{P}} \sum_{i \in E^{P}}\left(a_{i i^{\prime}}+a_{i^{\prime} i}\right) t_{i j}\left(1-t_{i j}\right) \leq \hat{P}_{j}, \quad j \in J,
$$

here $\hat{P}_{j}$ - the capacity of the terminal $j$ allowing to service/ handle goods in the period of time $\tau_{m}$.

Algorithmic restrictions identified for

- the coefficient of carrying capacity

$$
k_{k}\left(P_{k}, N_{A}, A^{g}, D, N_{j}^{p r m}\right)>k_{k}^{n}
$$

- the coefficient of run exploitation

$$
k_{r}\left(P_{k}, N_{A}, A^{g}, D, N_{j}^{p r m}\right) \geq k_{r}^{n}
$$

- for the term of goods delivery

$$
T_{m}\left(P_{k}, N_{A}, A^{g}, D, N_{j}^{p r m}\right) \leq T^{n} ;
$$

- the time of goods stay in the warehouse

$\tau_{m j}\left(P_{k}, N_{A}, A^{g}, D, N_{j}^{p r m}\right) \leq \tau^{n}, \forall m \in M ;$

- average technical speed of road vehicles

$$
V_{\text {tech. }}\left(P_{k}, N_{A}, A^{g}, D, N_{j}^{p r m}\right) \leq V_{\max }^{n},
$$

here $K_{k}$ and $K_{k}^{n}$ - real and normative coefficients of carrying capacity; $K_{r}$ and $K_{r}^{n}-$ real and normative coefficients of run exploitation; $T_{m}$ and $T_{m}^{n}$ - real and normative terms of delivery of goods; $M$ - set of indices of goods consignments; $V_{\text {tech. }}$ and $V_{\text {tech. }}^{n}$ - real and normative average technical speed of road vehicles; $\tau_{m j}$ - real time of the consignment $m$ goods stay in the $j$ warehouse; $\tau^{n}$-normative time of stay of goods in the warehouse; $N_{A}$-set of vehicles characteristics (number of vehicles, their owner dependence, etc.); $A^{g}-$ the matrix of freight flows of the region, $A^{g}=\left\|a_{i^{\prime}}\right\| ; D-$ the matrix of distances between the points in the region, $D=\left\|d_{i i}\right\| ; N_{j}^{p r m}$ - the number of handling places in the warehouse $j$.

Thus the problem $a$ - the distribution of points of the regional set into the servicing zones of the warehouses - is solved by the minimisation of the final function (16), when there are constraints (17)-(28).

For the formalisation of the problem $b$ the term overall freight operation is introduced for the incoming and outgoing goods into the zone of the warehouse $j$ established at the point $i$. It is defined as follows:

$$
\begin{aligned}
& \beta_{i j}=\sum_{i^{*} \in E^{P}} \sum_{i^{\prime} \in E^{P}}\left(a_{i^{*} i^{\prime}}+a_{i^{\prime} i^{*}}\right) d_{i i^{\prime}} t_{i^{\prime} j}\left(1-t_{i^{*} j}\right), \\
& j \in J_{k}, \quad i: b_{i}=1 .
\end{aligned}
$$

The problem $b$ is formulated as follows:

$$
\sum_{j \in J_{k}} \sum_{i \in E_{j}} \beta_{i j} y_{i j} \rightarrow \min
$$

$y_{i j}=\left\{\begin{array}{l}1, \text { if the warehouse } j \text { is established in the point } i \\ 0 \text { for the opposite case }\end{array}\right.$ 
when there are constraints of:

- the number of warehouses in each zone

$$
\sum_{i: b_{i}=1} y_{i j}=1, \quad j \in J_{k}
$$

- permissible rout distance between the warehouses in the region

$$
\begin{aligned}
& L_{\min } \leq d_{i i^{\prime}} y_{i j} y_{i^{\prime} j^{\prime}} \leq L_{\max }, \quad i \in E_{j}, i^{\prime} \in E_{j^{\prime}}, j, \\
& j^{\prime} \in J_{k} .
\end{aligned}
$$

As mentioned before, the model of the synthesis of the topological structure (16)-(28), besides the constraints (17)-(23), (30)-(31), which are the analytical functions of the structural elements of the system, comprises also the constraints (24)-(28), which are complex functions of structural and functional characteristics and are defined in the algorithmic form. These constraints indicate essential dynamic aspects of the projected systems and its elements functioning and they can be defined by the way of simulation modelling of discrete developments.
Thus the model of the analysed structure synthesis will be as follows:

$$
F(\bar{X}) \rightarrow \operatorname{extr}, \bar{X} \in \sigma,
$$

here $\sigma=\bar{\sigma} \cap \overline{\bar{\sigma}}, \bar{\sigma}, \overline{\bar{\sigma}}$

- the set of structure variants allowed according to the analytical and algorithmic constraints.

The optimisational simulation procedure was developed enabling the use of optimisation and simulation models jointly for the search of analytical variants of the structure.

Below certain data of modelling results are presented.

The results obtained by the way of modelling are presented in Table 1 and Table 2 and in Figs 1, 2, 3, and 4. These data enable to gauge the impact of different structural characteristics of the system on its functioning thus improving the quality of the project solutions at the stage of the synthesis of the system structure.

The size of goods consignment is a random quan-

Table 1. Distribution laws of stochastic variable meanings

\begin{tabular}{|l|c|c|c|c|c|}
\hline \multirow{2}{*}{\multicolumn{1}{|c|}{ Stochastic variable }} & \multirow{2}{*}{$\begin{array}{c}\text { Distribution } \\
\text { law }\end{array}$} & & \multicolumn{3}{c|}{ Parameters of distribution law } \\
\cline { 3 - 6 } & & Average & $\begin{array}{c}\text { Least } \\
\text { meaning }\end{array}$ & $\begin{array}{c}\text { Highest } \\
\text { meaning }\end{array}$ & $\begin{array}{c}\text { Standard } \\
\text { digression }\end{array}$ \\
\hline Type of goods & Uniform & 2,5 & 1,0 & 4,0 & - \\
\hline Size of goods consignment & Puasson's & 7,0 & 1,0 & 25,0 & - \\
\hline Time of delivery of goods consignment & Puasson's & 12,0 & 0 & 24,0 & - \\
\hline Duration of vehicle handling & Normal & 1,0 & 0,8 & 1,15 & 0,15 \\
\hline Vehicle traffic speed & Normal & 50,0 & 45,0 & 55,0 & 5,0 \\
\hline Number of goods consignment delivered to the warehouse No 1 & Puasson's & 80,0 & 40,0 & 200,0 & - \\
\hline Number of goods consignment delivered to the warehouse No 2 & Puasson's & 60,0 & 30,0 & 140,0 & - \\
\hline Number of goods consignment delivered to the warehouse No 3 & Puasson's & 60,0 & 30,0 & 140,0 & - \\
\hline Number of goods consignment delivered to the warehouse No 4 & Puasson's & 130,0 & 90,0 & 350,0 & - \\
\hline
\end{tabular}

Table 2. Results obtained by modelling

\begin{tabular}{|l|c|c|c|c|}
\hline \multirow{2}{*}{} & \multicolumn{3}{|c|}{ Names of locations of warehouses } \\
\cline { 2 - 5 } & Variables & Kaunas & Klaipèda & Siauliai \\
\hline Quantity of consigned goods, thousand $\mathrm{t}$ & 62,4 & 40,0 & 52,7 & 56,2 \\
\hline Quantity of outgoing goods, thousand t & 58,7 & 38,1 & 56,1 & 58,8 \\
\hline Amount of loaded vehicles & 694 & 557 & 460 & 640 \\
\hline Amount of consigned vehicles without goods & 71 & 118 & 120 & 54 \\
\hline Amount of unloaded vehicles & 563 & 441 & 487 & 564 \\
\hline Average loading coefficient & 0,64 & 0,54 & 0,72 & 0,65 \\
\hline Average time of goods stay in warehouse, $\mathrm{h}$ & 64,2 & 45,8 & 44,8 & 109,2 \\
\hline Average time of vehicle queuing, $\mathrm{h}$ & 0 & 0 & 0,53 & 0,09 \\
\hline Average number of queuing vehicles & 0 & 0 & 0,42 & 0,04 \\
\hline Maximum number of queuing vehicles & 0 & 0 & 3,0 & 1,0 \\
\hline
\end{tabular}




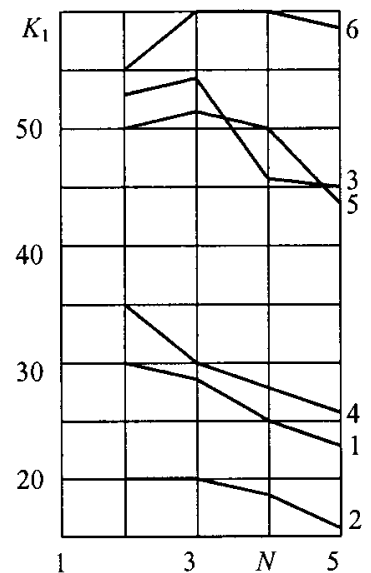

Fig 1

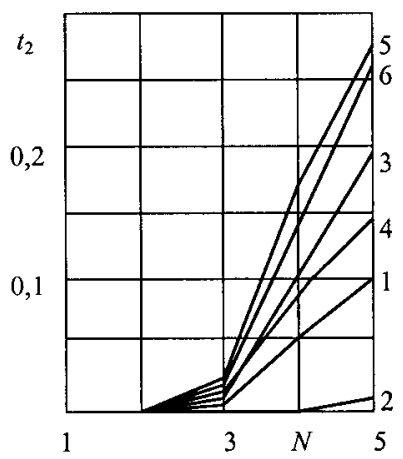

Fig 3

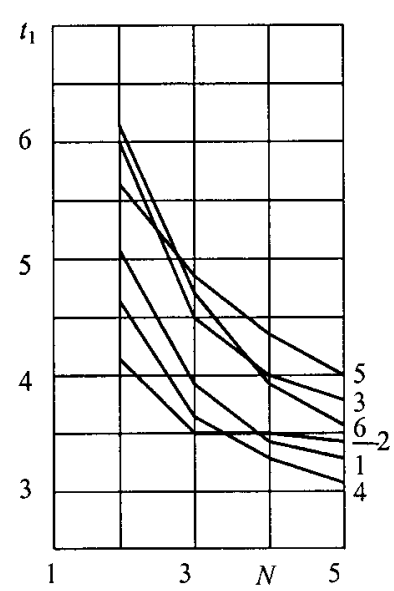

Fig 2

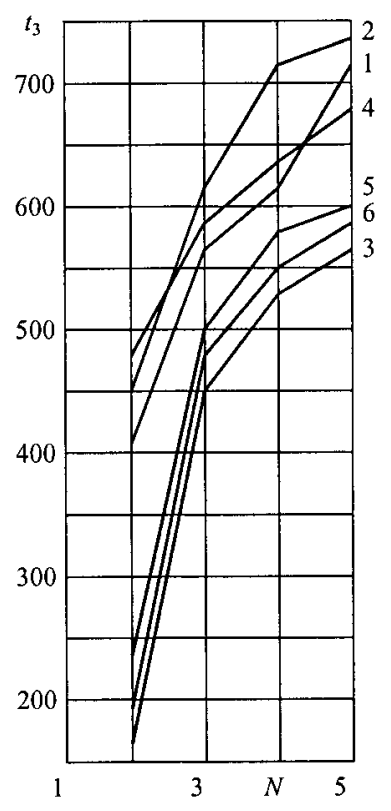

Fig 4 tity distributed according to the law with mathematic probability of $0,5 \mathrm{t}$ and with a standard digression of $0,1 \mathrm{t}$. Average speed of the vehicle is a normal random quantity with the mathematic probability of $50 \mathrm{~km} / \mathrm{h}$ and with a standard digression of $1,7 \mathrm{~km} / \mathrm{h}$.

The formulated problem model concerning the distribution of freight flows in the transport terminal to the consumers enables gauging the vehicle possibilities (dimensions, carrying capacity), limitations of delivery time, the time resources of a vehicle and general costs.

\section{Conclusions}

1. The synthesis of the topological structure of the road freight transport regional (urban) system enables the evaluation of impact on the functioning of the system, which is exercised by various structural system characteristics, thus improving the quality of project solu- tions at the stage of synthesis of the system structure.

2. Optimisation and simulation models included into the simulation procedure of topological structure optimisation have other possibilities as well. For example, the simulation model enables the solution of different problems related to the analysis of the system functioning, such as the investigation of system stability under the changes of its parametric meanings, also the comparative analysis of efficiency of different types of ruling impacts, of carrying, loading, handling technologies, etc.

3. The algorithm has been developed for the optimal definition by the carrying capacity of the structure of the vehicle fleet which enables the carrier to find a required average listing number of vehicles according to the carrying capacity.

4. The methods of the definition of an optimal size of the consignment of goods and the selection of a transportation type have been proposed, when the general storage and transportation costs are selected as the optimum criterion.

5. The algorithm has been developed for the optimisation of the local road network among the trade points. Testing of the algorithm proved that in the case when the density of the distribution of target functions meanings in the extremum zone is sufficiently large, the independent random search is sufficiently efficient even if compared with regular methods. Firstly, the number of the variants analysed is 200-500. Secondly, many variants close to the global extremum are found, which in most cases can be interpreted as an indeterminacy zone after later evaluation of incomprehensive initial information. Generally such a zone is found by the way of multi-critical optimisation with the application of regular methods.

\section{References}

1. Guilt J., Florian M., Crainic T.-G. A Multimode Multiproduct Network Assignment Model for Strategic Planning of Freight Flows. Transportation Science, Vol 24, No 1, February, 1990, p. 25-39.

2. Zhan F.-B., Noon Ch.-E. Shortest Path Algorithms: An Evaluation Using Real Road Networks. Transportation Science, Vol 32, No 1, February, 1998, p. 65-73.

3. Nuzzolo A., Russo F., Crisali U. A Doubly Dynamic Schedule - Based Assignment Model for Transit Network. Transportation Science, Vol 35, No 3, August, 2001, p. 268-285.

4. Bodin L., Mingozzi A., Baldacci R.; Ball M. The Roll onRoll off Vehicle Routing Problem. Transportation Science, Vol 34, No 3, August, 2000, p. 256-271.

5. Baublys A. Optimization of Freight Reloading at the Terminal. Transport Engineering (Transportas), Vol XIII, No 3, Vilnius: Technika, 1998, p. 88-92 (in Lithuanian). ISSN 1648-4142.

6. Baublys A. A Model of the Efficiency Analysis of Road Transport and Port Interaction. Transport Engineering (Transportas). Vol XVI, No 1, Vilnius: Technika, 2001, p. 8-10 (in Lithuanian). ISSN 1648-4142. 